\title{
Influence du stade de maturité de la plante de maïs récoltée pour ensilage sur la composition, la digestibilité apparente, les caractéristiques de fermentation dans le rumen et les performances zootechniques chez le taurillon à l'engraissement
}

\author{
AP Mayombo *, I Dufrasne, JL Hornick, M Diez, L Istasse \\ Nutrition animale, faculté de médecine vétérinaire, université de Liège, \\ B 43, Sart-Tilman, 4000 Liège, Belgique
}

(Reçu le 25 janvier 1996 ; accepté le 22 août 1996)

\begin{abstract}
Summary - Influence of the maturity stage of whole maize plant harvested for silage on the composition, apparent digestibility, fermentation characteristics in the rumen and animal performances in growing fattening bulls. Maize was harvested as whole plant to three different stages of maturity (S1, S2, S3) corresponding to dry matter contents of $25.1 \%$ (S1), 29.8\% (S2), 32.3\% (S3). The yields were, respectively, 10.7, 11.1 and 11.8 tons dry matter per ha. Each of these maize silages, offered to growing fattening bulls, was supplemented with dried sugar beet pulp, soya bean meal, minerals and vitamins. The stage of maturity did not affect ash, insoluble ash, ether extract or protein contents; however, a significant reduction of the fibre content was recorded with the late stage (S3). The stage of maturity did not affect apparent digestibility of dry matter and organic matter either in the whole diet or in the maize silage fraction measured in vivo on bulls maintained in metabolism stalls. In contrast, the digestibility of fiber was significantly reduced (62.1 vs 67.2 or $65.6 \%$ in the diet; 54.1 vs 63.3 or $60.7 \%$ in maize silage). In terms of rumen potentiality to degrade the feedstuffs, it was noted that total degradability of the different feedstuffs incubated was greater with the maize silage with a high dry matter content. There were no significant effects of the stage of maturity on animal performances during the ad libitum feeding trial lasting 5.5 months. From the feed conversion ratio, the total live weight gain and the yield of dry matter per ha, it was possible to estimate that $13.8,14.2$ and 15.7 bulls can be fattened, respectively, when maize was harvested in a early, medium or later stage of maturity.
\end{abstract}

maïze silage / stages / digestibility / fermentation / performance / bull

* Correspondance et tirés à part.

Fax : (32) 43664122 ; courriel : mayombo@stat.fmv.ulg.ac.be 
Résumé - Un maïs plante entière a été ensilé à trois stades de maturité différents ( $\mathrm{S} 1, \mathrm{~S} 2, \mathrm{~S} 3)$ correspondant à des teneurs respectives en matière sèche de 25,1 (S1), 29,8 (S2) et 32,3\% (S3). La production de matière sèche s'élevait à 10,$7 ; 11,1$ et 11,7 tonnes par hectare. Chacun de ces ensilages, distribué à des taureaux à l'engraissement, a été supplémenté par des pulpes séchées, du tourteau de soja, des sels minéraux et des vitamines. Le stade de maturité n'a pas affecté les teneurs en cendres, cendres insolubles, extrait éthéré ou protéines ; cependant une réduction significative de la teneur en fibre ADF a été enregistrée avec S3. La digestibilité de la matière sèche et de la matière organique de la ration mesurée in vivo sur des taureaux en cages à bilan ainsi que celle du maïs ensilage n'ont pas été non plus influencées par le stade de maturité. En revanche, la digestibilité de la fibre $\mathrm{ADF}$ a été significativement plus faible pour $S 3$ par rapport à $S 1$ et $S 2(62,1$ vs 67,2 ou $65,6 \%$, $p<0,05$ pour la ration; 54,1 vs 63,3 ou $60,7 \%, p<0,001$ pour le maïs). Quant aux potentialités du rumen à dégrader les aliments, il est à noter que la dégradabilité totale par la méthode in sacco des différents composants testés a été supérieure dans le rumen des animaux qui recevaient le maïs à teneur en matière sèche élevée. Il n'y a pas eu d'effets significatifs du stade de maturité sur les performances animales durant un essai de 5,5 mois dans le cas d'une alimentation ad libitum. À partir de l'indice de consommation, du gain total de poids vif et de la production de matière sèche par hectare, il était possible d'estimer que 13,8,14,2 et 15,7 taurillons pouvaient être engraissés respectivement à partir des ensilages de maïs $\mathrm{S} 1, \mathrm{~S} 2$ et $\mathrm{S} 3$ produits par ha.

\section{ensilage maïs / stade / digestibilité / fermentation / performance / taurillon}

\section{INTRODUCTION}

L'ensilage de maïs est très largement utilisé comme aliment de base pour les vaches laitières et les taureaux à l'engraissement. Ces derniers nécessitent une densité énergétique très élevée dans la ration. Or il existe des écarts de digestibilité importants entre les variétés de maïs Ascot, Gracia, Julius et Lixis (Istasse et al, 1990). Ces écarts peuvent entraîner des différences appréciables dans les performances de bovins à l'engraissement (Gielen et al, 1988 ; Gielen et al, 1990 ; Istasse et al, 1990 ; Barrière et al, 1995). Le maïs se caractérise, par rapport aux fourrages traditionnnels, par la juxtaposition en parties presque égales de grains et d'une partie non grain composée de tiges, de feuilles, de spathes et de rafles. Le stade de maturité de la plante de maïs à la récolte est un des critères qui détermine la valeur nutritive de l'ensilage (Aseltine, 1988 ; Flachowsky et al, 1993 ; Demarquilly, 1994) et, par conséquent, pourrait influencer les performances des animaux.

Le présent article a pour but de décrire les effets du stade de maturité du maïs sur la com- position chimique, la digestibilité apparente, la cinétique de dégradation dans le rumen, les paramètres de fermentation dans le rumen et les performances zootechniques chez les taurillons à l'engraissement.

\section{MATÉRIEL ET MÉTHODES}

\section{Culture}

Six ha d'une même parcelle de terre de culture ont été préparés suivant les techniques culturales habituelles. La fumure de base était constituée de 140 unités de phosphore, de 220 unités de potassium et de 122 unités d'azote par hectare. La variété de maïs Alarik (LG 2080) a été semée à la densité de 102000 pieds par hectare. Un traitement contre les adventices a été effectué avec $5 \mathrm{~L}$ de Laddok et 1,5 L d'huile par hectare. La récolte a été réalisée 124 jours après le semis pour le stade I (S1), 131 jours pour le stade 2 (S2) et 153 jours pour le stade 3 (S3). L'ensilage a été effectué en silo taupinière sur une dalle de béton. Les silos ont été ouverts environ 2,5 mois après leur réalisation. 


\section{Animaux}

Au total, 24 taureaux Blanc Bleu Belge de type culard en croissance engraissement et répartis en trois groupes, soit huit animaux par type d'ensilage de maïs, ont été placés en stalles à métabolisme pendant toute la durée de l'essai. Six de ces taureaux, soit deux par type d'ensilage de maïs étaient munis d'une canule du rumen en vue d'étudier les caractéristiques de fermentation et la dégradabilité dans le rumen.

\section{Rations}

L'ensilage de maïs représentait $60 \%$ des apports en matière sèche (MS) de la ration. Le tourteau de soja utilisé comme source de matières azotées a été distribué en quantité constante pendant l'engraissement à raison de $1,55 \mathrm{~kg}$ par jour. En vue de diversifier les glucides de la ration, de la pulpe séchée représentait le reste des apports. Un complexe minéral vitaminé ( $\mathrm{Ca}: 16 \%$, $\mathrm{P}: 8 \%$ ), distribué en quantité constante, soit 120 $\mathrm{g}$ par taurillon et par jour, corrigeait les insuffisances en minéraux et oligoéléments. Les quantités d'aliments distribués en deux repas étaient ajustées tous les 15 jours en vue d'un rationnement proche de l'ad libitum.

\section{Mesures}

Les quantités récoltées ont été pesées le jour de la réalisation des ensilages. Des échantillons d'ensilage ont été prélevés toutes les 2 semaines lors du désilage en vue de déterminer la composition chimique. Deux fractions, les grains d'une part et les tiges et les feuilles d'autre part, ont été obtenues par triage manuel à partir des trois ensilages. Ils ont été séchés dans une étuve ventilée maintenue à $50^{\circ} \mathrm{C}$ jusqu'à poids constant. La dégradation dans le rumen de dix aliments de composition chimique très variable a été étudiée. Il s'agissait de tourteau de soja (TS), de pulpes séchées (PS), d'orge (O), de maïs grain sec (MG), de grains de l'ensilage de maîs $S 1, S 2$ et S3; de tiges et feuilles de l'ensi- lage de maïs S1, S2 et S3. L'incubation de l'O, du MG et des grains de l'ensilage S1, S2 et S3 a permis de déterminer l'activité amylolytique dans le rumen. Les potentialités de dégradation des fibres ont été mesurées à partir de l'incubation des tiges et feuilles de l'ensilage S1, S2 et S3 ainsi que des PS. L'activité protéolytique a été comparée à partir du TS. Tous les aliments ont été broyés dans un broyeur à marteaux muni d'une grille de $1,0 \mathrm{~mm}$. La dégradabilité des aliments a été déterminée par la technique des sachets de nylon décrite par Mehrez et Ørskov (1977). Les sachets de nylon $(12 \times 7,5 \mathrm{~cm}$ de taille, $63 \mu$ de porosité), remplis de 5 à $6 \mathrm{~g}$ d'aliments concentrés ou de 3 à $4 \mathrm{~g}$ d'aliments grossiers ont été incubés pendant 4, 8, 16, 24 ou 48 heures dans le rumen. Les mesures ont été repétées quatre fois. La dégradation moyenne de la MS obtenue aux différents temps d'incubation a été utilisée pour calculer les paramètres de la cinétique de disparition de la MS selon l'équation : $d=a+b\left(1-\mathrm{e}^{-c t}\right)(\emptyset$ rskov et Mc Donald, 1979).

La dégradabilité théorique (DT) a été calculée à partir de l'équation proposée par $\mathrm{Mc}$ Donald (1981): DT $=a+(b c / c+k)$ où $k$ est le taux de sortie des particules du rumen estimé à $6 \%$ par heure (Vérité et al, 1987). Les paramètres de fermentation ont été déterminés sur des échantillons prélevés au moyen d'un tube muni d'une crépine. Huit échantillons ont été prélevés à intervalle de 2 heures, le premier échantillon étant prélevé avant le repas du matin. Le second repas a été distribué 8 heures après le premier repas. Le dernier échantillon a été prélevé 6 heures après le deuxième repas. Une solution de thiomersal à $3 \%$ a été ajoutée au liquide après le prélèvement en vue de bloquer les fermentations. Le liquide a ensuite été filtré sur de la gaze. Le pH a été déterminé immédiatement après le prélèvement à l'aide d'un potentiomètre à électrode $\mathrm{Ag} / \mathrm{AgCl}$ et les échantillons ont été stockés au congélateur en vue de la détermination de l'ammoniaque, du glucose et des acides gras volatils.

Les consommations ont été mesurées individuellement et quotidiennement. La digestibi- 
lité de la ration a été déterminée pour chaque coupe d'ensilage par récolte totale des matières fécales pendant une période de 8 jours. La digestibilité des composants de l'ensilage a été obtenue par soustraction (Schneider et Flatt, 1975) en se basant sur les coefficients de digestibilité proposés par Inra (1988) pour les autres composants de la ration ( pulpes séchées et tourteau de soja).

Les animaux ont été pesés tous les 28 jours et lors de leur départ pour l'abattoir. L'abattage a été décidé en fonction de l'état de finition des animaux estimé par maniement de la graisse sous-cutanée au niveau de la base de la queue, du pli du grasset et des côtes. Après abattage, le poids des carcasses a été déterminé et la composition des carcasses a été estimée à partir de celle du septième segment monocostal (Verbeke et Van De Voorde, 1978). Les données ont été traitées par analyse de la variance à un critère.

\section{Analyses chimiques}

La teneur en MS a été déterminée par dessication durant 24 heures à $105^{\circ} \mathrm{C}$, l'extrait éthéré par extraction à l'éther diéthylique et les cendres par calcination durant 24 heures à $550^{\circ} \mathrm{C}$. Les matières azotées $(\mathrm{N} \times 6,25)$ ont été déterminées par la méthode de Kjeldahl sur un Block Digestor de Tecator et la lignocellulose sous forme d'Acid Détergent Fiber (ADF) selon la méthode de Van Soest (1963). Le dosage de l'ammoniaque dans les digestats a été réalisé par la méthode de Berthelot adaptée à l'Autoanalyser de Technicon (Van Eenaeme et al, 1969). Quant au glucose, il a été dosé par la méthode à l'orthotoluidine sur autoanalyser (Charlier et al, 1974). L'amidon contenu dans les grains de maïs a été dosé par la méthode enzymatique à l'amyloglucosidase et l'analyse du glucose par la méthode colorimétrique automatisée au Technicon (Charlier et al, 1974). Les minéraux Ca et $\mathrm{Mg}$ et les oligoéléments $\mathrm{Cu}, \mathrm{Zn}, \mathrm{Mn}$ et $\mathrm{Fe}$ ont été dosés par spectrométrie d'absorption atomique; le $\mathrm{Na}$ et le $\mathrm{K}$ par émission de flamme. La concentration en $\mathrm{P}$ a été déterminée par colorimétrie au vanado-molybdate. Les acides gras volatils ont été déterminés par chromatographie en phase gazeuse (Van Eenaeme et al, 1965).

\section{RÉSULTATS}

La production de matière fraîche de maïs plante entière aux trois stades de récolte a été de 42,9 , 37,1 et 35,9 tonnes respectivement pour S1, S2 et $\mathrm{S} 3$ avec des teneurs en MS significativement différentes $(p<0,001): 25,1,29,8$ et $32,3 \%$, de sorte que la production de MS s'est élevée respectivement à 10,7,11,1 et 11,7 tonnes par hectare. La composition chimique de la plante de maïs aux trois stades est donnée dans le tableau I. Les teneurs en cendres, en cendres insolubles et en extrait éthéré sont faibles. La teneur en matières azotées est de $7,2 \%$. Ces teneurs ne sont pas modifiées par la date de récolte. En revanche, la teneur en ADF diminue ( $S 1$ vs $S 3$, $p<0,001$ et $\mathrm{S} 1$ vs $\mathrm{S} 2, p<0,05)$. À l'opposé, la teneur en amidon augmente avec le stade de maturité, la différence est significative au seuil $p<0,001$. Les teneurs en minéraux et en oligoéléments sont très proches dans les différents ensilages.

Les coefficients de digestibilité apparente de la ration et de l'ensilage sont repris dans le tableau II. Il n'y a pas eu d'effets significatifs du stade de récolte sur la digestibilité de la MS ( 78,7 et $76,8 \%$ pour la ration et l'ensilage respectivement) et de la matière organique $(80,1$ et $77,5 \%$ ). En revanche, la digestibilité des fibres est plus faible pour $\mathrm{S} 3$ : pour la ration, elle est de 62,1 vs 67,2 ou $65,6 \%(p<0,05)$; la différence étant plus importante pour la digestibilité de l'ADF de l'ensilage du maïs calculée par différence $(54,1$ vs 63,3 ou $60,7, p<0,001)$.

Les paramètres décrivant la cinétique de dégradation dans le rumen sont donnés dans les tableaux III et IV. Lorsqu'on s'intéresse, toute ration confondue, à la dégradabilité théorique des tiges et des feuilles, il apparaît de façon paradoxale que c'est pour le maïs récolté précocement que cette dégradabilité est la plus faible $(p<0,001)$. En revanche, pour les grains, c'est 
Tableau I. Composition chimique de la plante de maïs lors de la récolte à trois stades différents de maturité.

\begin{tabular}{|c|c|c|c|c|c|}
\hline & $S 1$ & $\$ 2$ & $S 3$ & $S E D$ & Seuil de signification \\
\hline Matière sèche (\%) & $25,1^{\mathrm{c}}$ & $29,8^{\mathrm{b}}$ & $32,3^{\mathrm{a}}$ & 1,10 & 0,001 \\
\hline \multicolumn{6}{|l|}{$E n \% M S$} \\
\hline Cendres & 3,9 & 3,7 & 3,8 & 0,34 & NS \\
\hline Cendres insolubles & 1,0 & 0,7 & 0,7 & 0,22 & NS \\
\hline Matière organique & 96,1 & 96,3 & 96,2 & 0,34 & NS \\
\hline ADF & $31,1^{\mathrm{a}}$ & $26,9^{b}$ & $24,1 \mathrm{bc}$ & 1,82 & 0,01 \\
\hline Extrait éthéré & 3,9 & 3,8 & 3,8 & 0,35 & NS \\
\hline Protéine brute & 7,3 & 7,2 & 7,2 & 0,33 & NS \\
\hline Extractif non azoté & $53,8^{\mathrm{c}}$ & $58,4^{\text {ab }}$ & $61,2^{\mathrm{a}}$ & 1,70 & 0,001 \\
\hline Amidon & $21,0^{c}$ & $30,5^{\mathrm{ab}}$ & $33,0^{\mathrm{a}}$ & 1,14 & 0,001 \\
\hline \multicolumn{6}{|l|}{ En $\mathrm{g} / \mathrm{kg} M S$} \\
\hline Phosphore & 1,1 & 1,1 & 1,2 & 0,12 & NS \\
\hline Calcium & 2,3 & 2,4 & 2,5 & 0,49 & NS \\
\hline Sodium & 0,3 & 0,2 & 0,2 & 0,04 & NS \\
\hline Potassium & 10,2 & 10,1 & 10,4 & 0,73 & NS \\
\hline Magnésium & 1,0 & 1,0 & 1,2 & 0,08 & NS \\
\hline \multicolumn{6}{|l|}{$E n m g / k g M S$} \\
\hline Cuivre & 9,3 & 12,4 & 6,3 & 5,81 & NS \\
\hline Zinc & 127,9 & 130,0 & 166,7 & 52,25 & NS \\
\hline Manganèse & 22,9 & 21,4 & 28,4 & 7,48 & NS \\
\hline Fer & 501,4 & 333,7 & 354,9 & 76,47 & NS \\
\hline
\end{tabular}

S1 : stade $1 ; \mathrm{S} 2:$ stade $2 ; \mathrm{S} 3:$ stade 3 ; les valeurs suivies de lettres différentes sont significativement différentes. NS : non significatif au seuil de $p>0,05$.

avec S1 que la dégradabilité est la plus grande. Enfin, la dégradabilité du maïs grain sec est de loin inférieure à celle des grains des ensilages ou du grain d'orge $(p<0,001)$. Pour tous les aliments incubés à l'exception de l'O, la fraction rapidement dégradable est supérieure avec la ration contenant l'ensilage SI. De manière générale, la dégradabilité totale est plus grande pour les différents aliments incubés avec la ration ensilage $\mathrm{S} 3$; certaines différences sont significatives pour les temps d'incubation longs notamment pour le grain de l'ensilage $S 1(p<0,001)$, S2 $(p<0,001)$ et S3 $(p<0,03)$, Ia pulpe $(p<0,04)$ et le maïs grain sec $(p<0,05)$.

Les paramètres relatifs aux fermentations dans le rumen sont présentés dans la figure 1.
Le $\mathrm{pH}$ moyen obtenu sur huit prélèvements est de 6,6 pour $S 1,6,9$ pour $S 2$ et 6,8 pour $S 3$; les différences sont significatives $(p<0,05)$. Les concentrations en acides gras volatils totaux sont de 118,$0 ; 97,6$ et $101,5 \mathrm{mmol} / \mathrm{L}$ respectivement pour les stades 1-2-3; les différences sont également significatives $(p<0,01)$. La concentration en ammoniaque est quelque peu plus élevée avec $\mathrm{S} 1(96,1 \mathrm{mgN} / \mathrm{L})$ que $\mathrm{S} 3(80,0 \mathrm{mgN} / \mathrm{L})$ et $\mathrm{S} 2$ (76,0 mgN/L). Il en est de même pour la concentration en glucose soit $1046 \mathrm{mg} / \mathrm{L}$ pour S1, 906,4 $\mathrm{mg} / \mathrm{L}$ pour $\mathrm{S} 3$ et $721,2 \mathrm{mg} / \mathrm{L}$ pour S2. L'évolution en fonction du temps de la concentration en acides gras volatils totaux est classique : valeur faible avant le premier repas, augmentation pour atteindre un maximum deux heures 
Tableau II. Digestibilité apparente des rations et de l'ensilage de maïs récolté à trois stades différents de maturité (\%).

SI

\section{Digestibilité de la ration \\ Matière sèche \\ Matière organique \\ $\mathrm{ADF}$}

Digestibilité du maïs

Matière sèche

Matière organique

$\mathrm{ADF}$

\section{8,7}

80,1

$67,2^{\mathrm{a}}$

76,9

77,7

$63,3^{\mathrm{a}}$
$S 2$

79,3

80,5

$65,6^{\text {ab }}$

78,2

79,6

$62,1^{\mathrm{bc}}$

0,98

0,94

2,01

NS

NS

0,05

S1 : stade $1 ; \mathrm{S} 2$ : stade $2 ; \mathrm{S} 3:$ stade 3 ; les valeurs suivies de lettres différentes sont significativement différentes ; NS : non significatif au seuil de $p>0,05$.

après ce repas et ensuite diminution. La concentration augmente à nouveau après le deuxième repas, le maximum étant atteint 4 à 6 heures après ce dernier. Le profil de la concentration en glucose se caractérise par une augmentation progressive après le repas, les concentrations les plus élevées étant obtenues avec le maïs S1. La concentration en ammoniaque est élevée avant le repas du matin; elle descend pour atteindre des valeurs basses 4 à 6 heures après ce dernier. Une évolution semblable apparaît après le second repas.

Le poids initial moyen des taurillons était de $323 \mathrm{~kg}$ tandis que le poids final est de $537 \mathrm{~kg}$, soit un gain de poids vif moyen de $1,32 \mathrm{~kg}$ par jour. Il n'y a pas de différences significatives

Tableau III. Caractéristiques de dégradation dans le rumen ( $a, b, c$ et DT) de la matière sèche d'aliments riches en amidon ou en fibres (données toute ration confondue).

Aliments riches en

\begin{tabular}{|c|c|c|c|c|c|c|c|c|}
\hline \multicolumn{3}{|c|}{ Amidon } & \multirow{3}{*}{$M G$} & \multirow{3}{*}{$O$} & \multicolumn{4}{|c|}{ Fibres } \\
\hline \multicolumn{3}{|c|}{ Grains maïs } & & & \multicolumn{3}{|c|}{ Tiges et feuilles mais } & \multirow[t]{2}{*}{$P S$} \\
\hline$S I$ & $S 2$ & $S 3$ & & & $S I$ & $S 2$ & S3 & \\
\hline 40,8 & 35,4 & 36,1 & 6,9 & 34,5 & 19,8 & 29,1 & 30,9 & 0,8 \\
\hline 58,0 & 64,0 & 61,1 & 93,1 & 51,8 & 49,9 & 43,3 & 55,8 & 93,0 \\
\hline 0,067 & 0,062 & 0,064 & 0,029 & 0,165 & 0,043 & 0,045 & 0,028 & 0,083 \\
\hline $71,4 \mathrm{ab}$ & $67,9 \mathrm{bc}$ & $67,6^{\mathrm{c}}$ & $37,2^{d}$ & $72,5 \mathrm{a}$ & $40,6^{c}$ & $47,7 \mathrm{~b}$ & $48,7^{b}$ & $54,8^{\mathrm{a}}$ \\
\hline
\end{tabular}

MG : maïs grain sec ; $\mathrm{O}$ : orge ; PS : pulpe séchée ; $\mathrm{S} 1$ : stade $1, \mathrm{~S} 2:$ stade $2, \mathrm{~S} 3$ : stade 3 ; les valeurs de DT des aliments de chaque groupe suivies de lettres différentes sont significativement différentes $(p<0,001)$. 


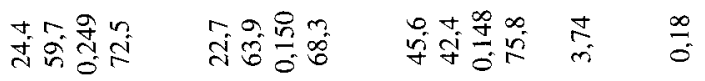

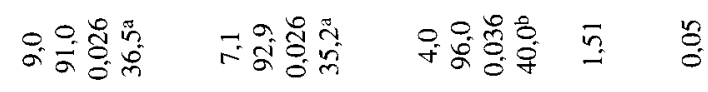

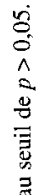

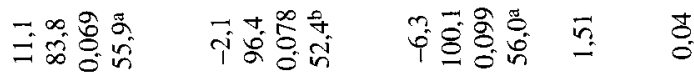

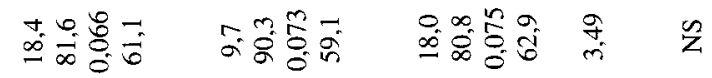

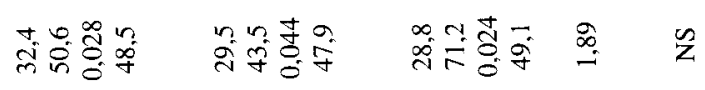

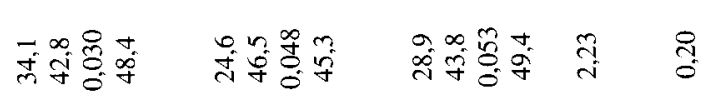

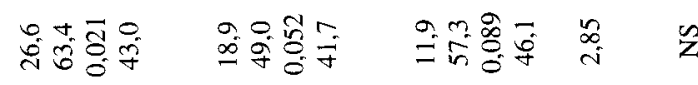

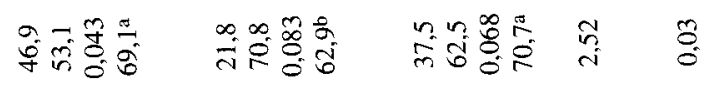

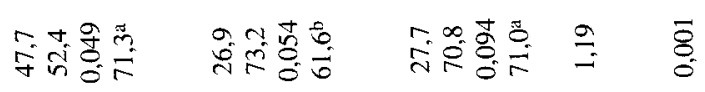

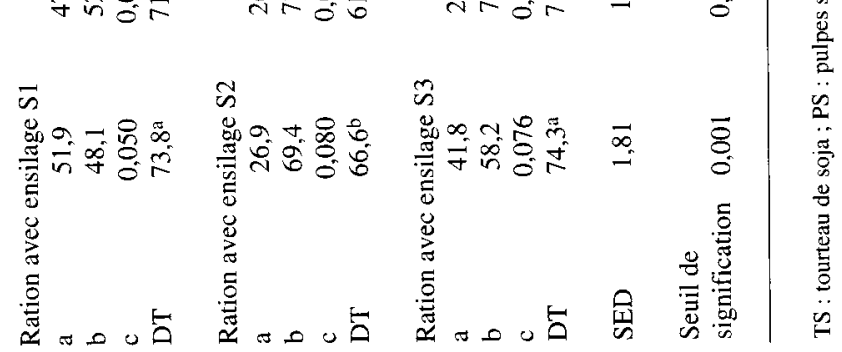



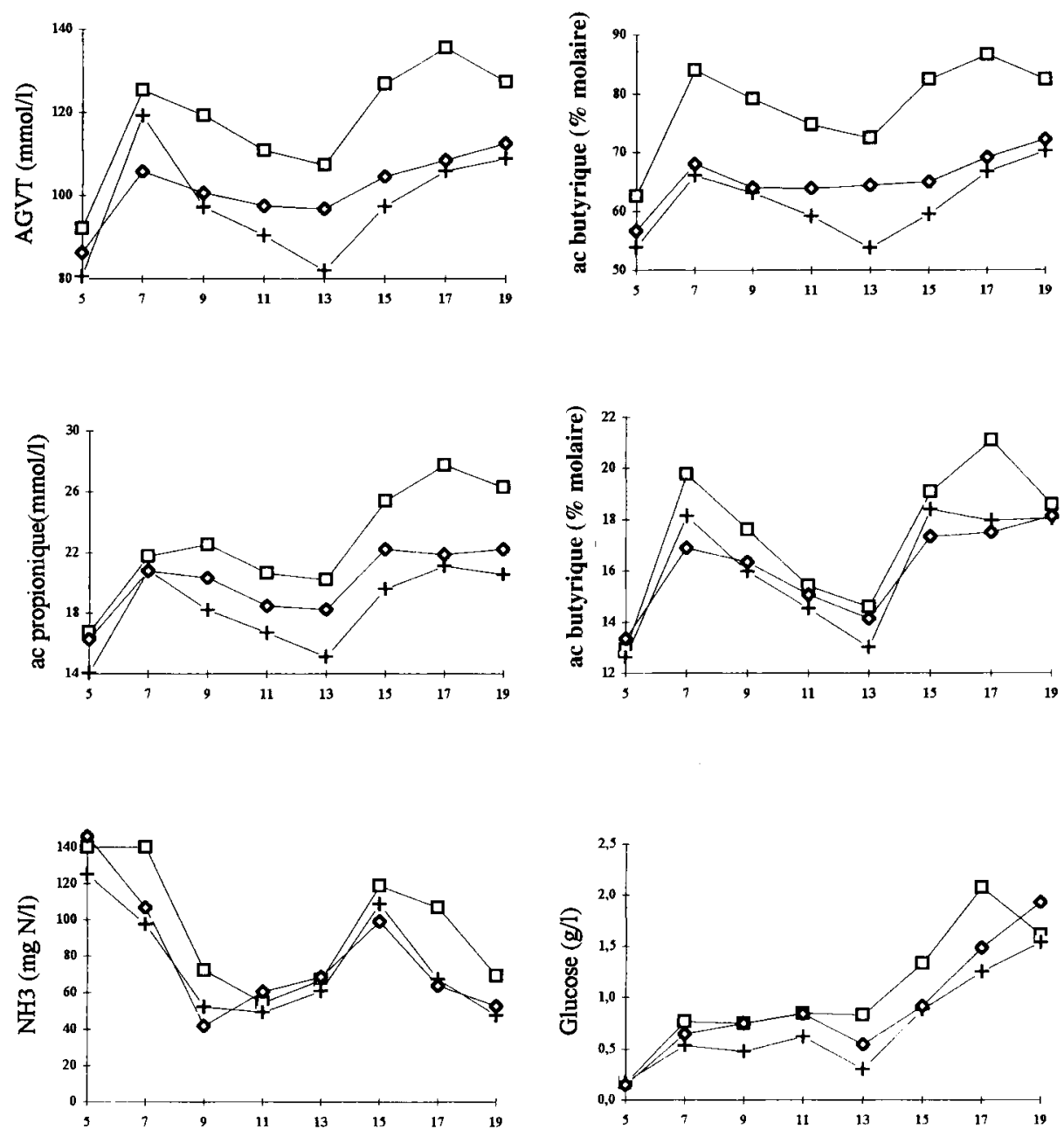

Fig 1. Paramètres de fermentation dans le rumen ; la ration étant distribuée à 5 et 13 heures.

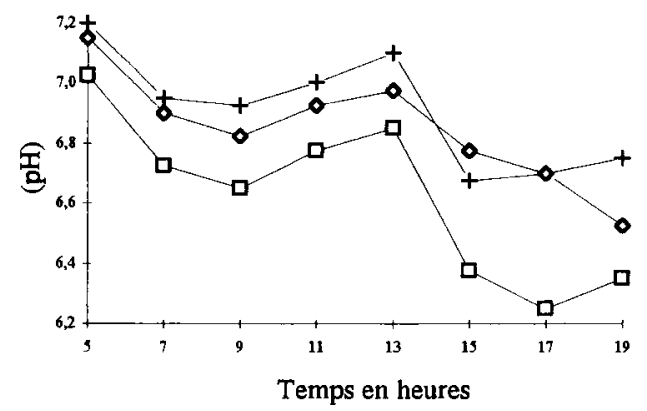


entre les groupes pour les paramètres mesurés (tableau V). Les consommations en $\mathrm{kg}$ de MS d'ensilage de maïs ont été de 4,8; 4,7 et $4,5 \mathrm{~kg}$ respectivement pour les stades 1,2 et 3 . La consommation totale est en moyenne de $7,63 \mathrm{~kg}$ de MS par jour et l'indice de consommation de $5,8 \mathrm{~kg}$ de MS / $\mathrm{kg}$ de croît. À l'abattage, les taurillons pèsent $525 \mathrm{~kg}$ et les carcasses $327 \mathrm{~kg}$, ce qui correspond à un rendement moyen de $62,4 \%$. Après dissection du segment monocostal et reconstitution de la carcasse, la proportion des muscles dans la carcasse désossée est de $85,7 \%$ et celle de tissu conjonctivo-adipeux de $14,3 \%$.

\section{DISCUSSION}

\section{Composition chimique}

Les faibles teneurs en cendres, cendres insolubles et fer indiquent que les opérations réalisées lors de l'ensilage ont été effectuées dans de bonnes conditions, sans contamination par de la terre. Une concentration minérale de $2,4 \mathrm{~g} / \mathrm{kg}$ de MS pour le $\mathrm{Ca}, 1,2 \mathrm{~g}$ pour le $\mathrm{P}, 0,24 \mathrm{~g}$ pour le $\mathrm{Na}$ et $1,1 \mathrm{~g}$ pour le $\mathrm{Mg}$ sont à considérer comme usuelles selon Cottyn et al (1984) et Inra (1988). Il en est de même pour les oligoéléments, mais il faut noter des teneurs élevées en zinc et faibles

Tableau V. Performances zootechniques de taurillons nourris avec une ration d'engraissement à base d'ensilage de maiis récolté à trois stades différents de maturité.

\begin{tabular}{|c|c|c|c|c|c|}
\hline & $S I$ & $S 2$ & $S 3$ & $S E D$ & $\begin{array}{l}\text { Seuil de } \\
\text { signification }\end{array}$ \\
\hline Nombre animaux & 8 & 8 & 8 & & \\
\hline \multicolumn{6}{|l|}{ Performances } \\
\hline Poids inital $(\mathrm{kg})$ & 331,1 & 309,5 & 328,0 & 34,30 & NS \\
\hline Poids final $(\mathrm{kg})$ & 543,4 & 518.4 & 547,9 & 28,23 & NS \\
\hline Durée (jour) & 161,9 & 160,1 & 163,6 & 8,21 & NS \\
\hline GQM (kg) & 1,31 & 1,30 & 1,34 & 0,07 & NS \\
\hline \multicolumn{6}{|l|}{ Consommations (kg MS/jour) } \\
\hline Ensilage de mäis & 4,8 & 4,7 & 4,5 & 0,48 & NS \\
\hline Tourteau de soja & 1,4 & 1,4 & 1,4 & - & NS \\
\hline Pulpes séchées & 1,5 & 1,5 & 1,7 & 0,25 & NS \\
\hline Total & 7,7 & 7,6 & 7,6 & 0,76 & NS \\
\hline \multicolumn{6}{|c|}{ Indice consommation ( $\mathrm{kg} \mathrm{MS} \mathrm{/} \mathrm{kg}$ croît) } \\
\hline Ensilage de maïs & 3,7 & 3,7 & 3,4 & 0,41 & NS \\
\hline Total & 5,88 & 5,85 & 5,67 & 1,14 & NS \\
\hline \multicolumn{6}{|l|}{ Données d'abattage } \\
\hline Poids abattage $(\mathrm{kg})$ & 528,0 & 507,9 & 538,9 & 27,50 & NS \\
\hline Poids carcasse $(\mathrm{kg})$ & 327,2 & 316,2 & 339,0 & 18,46 & NS \\
\hline Rendement (\%) & 62,1 & 62,4 & 62,8 & 1,79 & NS \\
\hline \multicolumn{6}{|l|}{ Carcasse désossée (\%) } \\
\hline Muscles & 86,4 & 84,9 & 85,9 & 1,42 & NS \\
\hline Tissu conjonctivo-adipeux & 13,6 & 15,1 & 14,1 & 1,42 & NS \\
\hline
\end{tabular}

S1 : stade 1, S2 : stade 2, S3 : stade 3; GQM : gain de poids vif moyen quotidien ; les valeurs moyennes ne sont pas significativement différentes entre elles $(p>0,05)$. 
en manganèse ; ces résultats sont en opposition avec ceux de l'Inra (1988). Cette inversion des concentrations en oligoéléments a déjà été rapportée antérieurement (Gielen et al, 1990) et serait à relier avec la nature du sol dans lequel le maïs est cultivé. La concentration en minéraux et en oligoéléments dans l'ensilage de maïs sont, de loin, en-dessous de la moyenne des besoins des taurillons en croissance-engraissement proposés par Guéguen et al (1988) et ont donc nécessité un supplément.

Le recul de la date de récolte a été accompagné d'une augmentation de la teneur en MS de l'ensilage. De manière générale, d'après une étude d'Andrieu et al (1993) portant sur 48 cultures de maïs différentes, l'augmentation de la teneur en MS avec l'âge résulte principalement d'une augmentation de la proportion de grain et de la teneur en MS des grains. De même, l'augmentation de la teneur en amidon avec le recul de la date de récolte est le résultat de la migration vers les grains des glucides solubles qui sont accumulés lors de la photosynthèse dans les tiges et feuilles.

\section{Digestibilité apparente}

Il n'y a pas eu d'effets importants du stade de récolte sur les digestibilités de l'ensilage de maïs pour la MS et la matière organique, ces coefficients sont en moyenne de 76,8 et 77,5\%. Ces digestibilités sont semblables à celles enregistrées antérieurement par notre groupe et rapportés par Gielen et al $(1988,1990)$ et Dufrasne et al (1991a). En revanche, elles sont environ de cinq unités plus élevées que la moyenne de $71,9 \%$ citée par Andrieu et al (1993). Pour Barrière et Émile (1992), la digestibilité de la matière organique est comprise entre 65 et $76 \%$, pour Demarquilly (1994), elle varie de 67 à $78 \%$ avec une valeur moyenne de $73 \%$. Le peu de différence concernant la digestibilité dans les résultats de l'expérience présente où la ration était distribuée à volonté corrobore les données rapportées par d'autres auteurs. Émile et al (1995) ont observé une digestibilité de la matière organique de $67,1 \%$ pour un maïs récolté à $28 \%$ de MS et de $68,5 \%$ pour le même maïs récolté à $35 \%$ de MS. Les valeurs rapportées par Pierre et al (1987) sont pour la digestibilité de la MS de $64,3 \%$ pour un maïs à teneur de $25 \%$ de MS, de $63,6 \%$ pour une teneur de $35,5 \%$ et de $61,2 \%$ pour $45,9 \%$ de MS. À l'opposé, Calder et al (1977) avaient observé au Canada de plus larges différences au niveau des coefficients de digestibilité en fonction de la teneur en MS ; il faut souligner cependant que certaines teneurs en MS étaient très faibles. Selon Demarquilly (1994), les différences de digestibilité pourraient être reliées non seulement au pourcentage de grains ou d'épis à un stade donné, mais également beaucoup plus à la différence dans la digestibilité des parois végétales. Il est important de noter que la diminution de digestibilité de la fibre observée dans cet essai est de 9,2 points soit $17 \%$ entre les stades S1 et S3.

\section{Cinétique de dégradation}

Toute ration ou tout stade de maturité confondus, la dégradation de la MS des grains de l'ensilage de maïs $\mathrm{S} 1$ a été supérieure à celle des grains des deux autres ensilages (tableau III). Ces derniers ont été néanmoins plus dégradables que les grains de maïs sec. Il est intéressant aussi de noter que la dégradabilité des grains des trois ensilages de maïs est pratiquement égale à celle de l'orge. Ces résultats sont à mettre en rapport tant avec le procédé de conservation (conservation sous forme humide ou sèche) qu'avec la structure macroscopique du grain d'amidon, qui évolue avec le stade de maturité. Si la structure de la molécule d'amidon et notamment les proportions d'amylose et d'amylopectine ont une influence sur la cinétique de dégradation, il faut aussi rappeler que la matrice protéique ainsi que les composants structuraux internes de la paroi entourant le grain d'amidon peuvent également jouer un rôle de protection de l'amidon et être responsables des différences de digestion entre l'orge et le maïs secs (Mc Allister et al, 1993). Mais il est vraisemblable que les différences de dégradabilité entre les grains des trois ensilages de maîs et les grains secs de maïs résultent sur- 
tout de la fragilisation de la matrice protéique durant l'ensilage. Paradoxalement, des résultats inverses sont observés lorsque la comparaison porte sur les tiges et feuilles. Les valeurs de dégradation sont de 40,$6 ; 47,7$ et $48,7 \%$ respectivement pour les tiges et feuilles des ensilages S1, S2 et S3. Dans les essais rapportés par Mir et al (1992) avec de l'ensilage de maïs récolté précocement ou tardivement, la dégradabilité de la MS a été respectivement de 51,8 et de $45,8 \%$ et la disparition après 72 heures d'incubation de l'ADF de 48,9 et de 43,5\%. Les résultats inverses observés dans l'expérience présente pourraient être dus au sèchage en étuve. Cette hypothèse est corroborée par les coefficients de digestibilité plus élevés pour l'ADF (63,3\% pour $\mathrm{S} 1$ vs $54,1 \%$ avec S3) (tableau II).

La fraction rapidement dégradable (a) ainsi que les dégradations aux courtes périodes d'incubation étaient particulièrement élevées avec la ration maïs $S 1$ (tableau IV) indiquant une rapide mise à disposition des nutriments pour les microorganismes du rumen. Les tendances pour une dégradabilité théorique plus élevée avec la ration contenant l'ensilage de maïs $S 3$, et ce pour l'ensemble des aliments incubés, suggèrent une activité de la flore du rumen supérieure et ce vraisemblablement pour les différents composants chimiques.

\section{Caractéristiques de fermentations}

Des teneurs élevées en ammoniaque avant le repas du matin suivies d'une réduction 4 à 6 heures après ce dernier ont déjà été observées antérieurement dans nos essais par Cordiez et al (1972) et par Van Eenaeme et al (1990). De tels profils sont en opposition avec ceux rapportés avec des rations à base de fourrage par Jarrige et al (1980) ou par Satter et Roffler (1981). Il est vraisemblable que les différences soient dues à un apport insuffisant de substrats fermentescibles avant le repas. Si tel est le cas, l'énergie est limitante pour la synthèse des protéines bactériennes ; l'azote n'étant pas incorporé, la concentration en ammoniaque est élevée. Après le repas, l'énergie est disponible pour la microflore ; l'azote est incorporé dans les protéines bactériennes et donc la concentration en ammoniaque diminue. La teneur plus élevée en glucose dans le liquide du rumen avec l'ensilage $\mathrm{S} I$ est probablement due à une solubilisation plus rapide des composants des grains et des tiges et feuilles.

\section{Performances zootechniques}

En moyenne, le gain quotidien a été de $1,32 \mathrm{~kg}$ et l'indice de consommation de $5,8 \mathrm{~kg}$ de $\mathrm{MS} / \mathrm{kg}$ de crô̂t. De telles performances sont considérées comme élevées avec une ration à base d'ensilage de maïs. En effet, Cottyn et al (1984) rapportent des gains de l'ordre de $1,1 \mathrm{~kg}$ et des indices de consommation de 7,1 kg MS/kg avec des rations à base d'ensilage de maiis complémentées par différentes sources de matières azotées. Les résultats rapportés par Malterre et al (1985) sur 46 et 47 animaux ont été de 1,27 et $1,46 \mathrm{~kg} / \mathrm{jour}$ et 6,63 et $6,12 \mathrm{~kg}$ MS $/ \mathrm{kg}$. Les données correspondantes avec différentes variétés de maïs ont été de 1,35 et $1,45 \mathrm{~kg} / \mathrm{jour}$ pour Gielen et al (1988) et de 1,33 $\mathrm{kg} / \mathrm{jour}$ pour Istasse et al (1990), les indices de consommation étant respectivement de 6,$66 ; 6,23$ et $6,08 \mathrm{~kg} \mathrm{MS} / \mathrm{kg}$. Il n'y a pas eu de différences entre les stades de récolte en ce qui concernent les performances animales individuelles dans le présent essai. Ces résultats confirme ceux rapportés par Dufrasne et al (1991b) lors d'une comparaison portant sur de l'ensilage de maïs récolté à 25,1 et 30,8\% de MS. Le peu de différence de la teneur en MS sur les performances avait déjà été rapporté par Calder et al (1977) pour certains paramètres. À partir de l'indice de consommation, du gain de poids total et de la production de MS, le nombre de taurillons engraissés par ha a été estimé à 13,8,14,2 et 15,7 respectivement pour l'ensilage S1, S2 et S3, soit une amélioration de $14 \%$ entre $S 1$ et $S 3$.

\section{CONCLUSION}

Cet essai montre que le recul de la date de récolte du maïs en vue de son ensilage sous forme de 
plante entière par période de 15 jours s'accompagne de différences importantes en terme de teneurs en MS, fibres et amidon. Des différences sont également observées au niveau de la digestibilité apparente de la fibre ainsi que du métabolisme dans le rumen. En revanche, il n'y a pas d'effets importants sur les performances en engraissement. Néanmoins, comme la quantité totale de MS récoltée est plus importante avec le recul de la date de récolte, il est possible d'engraisser plus d'animaux.

\section{REMERCIEMENTS}

Recherche subsidiée par l'Irsia-DGG, ministère fédéral de l'Agriculture.

\section{RÉFÉRENCES}

Andrieu J, Demarquilly C, Dardenne P, Barrière Y, Lila M, Maupetit P, Rivière F, Femenias N (1993) Composition and nutritive value of whole maize plants fed fresh to sheep. I. Factors of variation. Ann Zootech 42, 221-249

Aseltine MS (1988) Corn silage quality can vary depending on hybrid planted. Feedstuffs 60, 13-14

Barrière Y, Émile JC (1992) Variabilité génétique de la digestibilité du maïs ensilage mesurée sur des moutons standards. Genetic variation of in vivo digestibility and energetic feed value of maize silage. Inra Prod Anim 5, 247-255

Barrière Y, Émile JC, Argillier O, Hébert Y (1995) Effets du génotype de maïs ensilage sur les performances zootechniques de vaches laitières. Inra Prod Anim 8, 315-320

Calder FW, Langille JE, Nicholson JWG (1977) Feeding value for beet steers of com silage as affected by harvest dates and frost. Can J Anim Sci 57, 6573

Charlier C, Van Eenaeme C, Canart B, Pondant A, Lambot O, Bienfait JM (1974) Méthode de dosage semi-automatique de l'amidon et du glucose dans les aliments pour bétail. Ann Méd Vét 118, 181194

Cottyn BG, Boucqué CV, Fiems LO, Buysse FX (1984) L'utilisation d'ensilage de maiss dans la production intensive de viande bovine, Rev Agric 37, 649-660)
Cordiez E, Bienfait JM, Lambot O, Pondant A (1972) Le ravitaillement protéique des jeunes bovins en croissance-engraissement. In : Livre jubilaire dédié au prof A De Vuyst. Editorial Garsi, Madrid, 666690

Demarquilly C (1994) Facteurs de variation de la valeur nutritive du maïs ensilage. Inra Prod Anim $7,177-189$

Dufrasne I, Gielen M, Van Eenaeme C, Istasse L, Biston R, Bienfait JM (1991a) Influence of stage of maturity on chemical composition and digestibility of maize silage. In : Proceedings of the 42nd Annual Meeting of EAAP, RFA, Berlin, 338339

Dufrasne I, Istasse L, Gielen M, Midy G, Bienfait JM (1991b) Influence of stage of maturity of maize silage on animal performance. In : Proceedings of the 42th Annual Meeting of EAAP, RFA, Berlin, 424-425

Emile JC, Barrière Y, Traineau R (1995) Respective effects of genotype and dry matter content on maize silage feeding value. Ann Zootech 44, 55

Flachowsky G, Peyker W, Schneider A. Henkel K (1993) Fibre analyses and in sacco degradability of plant fractions of two corn varieties harvested at various times. Anim Feed Sci Tech 43, 41-50

Gielen M, Istasse L, Bienfait J M, Legros P (1988) Comparaison de deux variétés de mais ensilage en engraissement de taurillons. Les Élevages Belges 1, 37-39

Gielen M, Dufrasne I, Van Eenaeme C, Istasse L, Clinquart A, Bienfait JM, Biston R (1990) Ensilage de mais plante entière : comparaison de quatre variétés. I. Production, composition et digestibilité. Rev Agric 43, 987-994

Guégen L, Lamand M, Meschy F (1988) Nutrition minérale. In : Alimentation des bovins, ovins et caprins, Inra, Paris, 95-111

Inra (1988) Alimentation des bovins, ovins et caprins. Inra, Paris, $471 \mathrm{p}$

Istasse L, Gielen M, Dufrasne I, Clinquart A, Van Eenaeme C, Bienfait JM (1990) Ensilage de maïs plante entière : comparaison de quatre variétés. II Performances Zootechniques. Rev Agric 43, 9951005

Jarrige R, Journet M, Vérité R (1980) Azote. In : Alimentation des ruminants, Inra, Paris, 89-128

Malterre C, Bertin G, Gallais A, Huguet L, Micol D (1985) Le maïs brown midrib plante entière. 2. Utilisation sous forme d'ensilage par les taurillons. Bull Tech CRZV Inra 60, 43-58 
McAllister TA, Phillippe RC, Rode LM, Cheng KJ (1993) Effect of the protein matrix on the digestion of cereal grains by ruminal microorganisms. J Anim Sci 71, 205-212

Mc Donald I (1981) A revised model for the estimation of protein degradability in the rumen. $J$ Agric Sci Camb 96, 251-252

Mehrez AZ, Ørskov ER (1977) A study of the artificial fibre bag technique for determining the digestibility of feeds in the rumen. J Agric Sci Camb 88, 645-650

Mir Z, Mir PS, Bittman S, Fisher LJ (1992) Ruminal degradation characteristics of corn and corn-sunflower intercropped silages prepared at two stages of maturity. Can J Anim Sci 72, 881-889

Ørskov ER, McDonald I (1979) The estimation of protein degradability in the rumen from incubation measurements weighed according to rate of passage. J Agric Sci Camb 92, 499-503

Pierre NRS, Bouchard R, Laurent GS, Roy GL, Vinet C (1987) Performance of lactating dairy cows fed silage from corn of varying maturities. J Dairy Sci $70,108-115$

Satter LD, Roffler RE (1981) Influence of nitrogen and carbohydrate inputs on rumen fermentation. In : Recent Developments in Ruminant Nutrition (W Haresign, DJA Cole, eds), Butterworths. Londres, 115-139
Schneider BH, Flatt WP (1975) The Evaluation of Feeds through Digestibility Experiments. The University of Georgia Press, Athens, GA, 151-188

Van Eenaeme C, Bienfait JM, Lambot O (1965) La détermination quantitative des acides gras volatils dans le liquide du rumen par chromatographie en phase gazeuse. Ann Méd Vét 109, 569-584

Van Eenaeme C, Bienfait JM, Lambot O, Pondant A (1969) Détermination automatique de l'ammoniaque dans le liquide de rumen par la méthode de Berthelot adaptée à l'auto-analyser. Ann Méd Vét 113, 419-429

Van Eenaeme C, Istasse L, Gabriel A, Clinquart A, Maghuin RG, Bienfait JM (1990) Effects of dietary carbohydrate composition on rumen fermentation, plasma hormones and metabolites in growing fattening bulls. Anim Prod 50, 409-416

Van Soest PJ (1963) Use of detergents in the analysis of fibrous feeds. II. A rapid method for the determination of fiber and lignine. J Ass Off Agr Chem $46,829-835$

Verbeke R, Van De Voorde G (1978) Détermination de la composition de demi carcasses de bovins par la dissection d'une seule côte. Rev Agric 31, 875-880

Vérité R, Michalet-Doreau B, Chapoutot P, Peyraud JL, Poncet $C$ (1987) Révision du système des proteines digestibles dans l'intestin (PDI), Bull Techn CRZV, Inra 70, 19-34 